\title{
The Use of Collagen Hydrolysates and Native Collagen in Osteoarthritis
}

\author{
M Cristina de Almagro* \\ R\&D Department, Laboratorios Ordesa, Spain
}

*Corresponding author: M Cristina de Almagro, R\&D Department, Laboratorios Ordesa, Parc Científic de Barcelona, C/Baldiri Reixac 15-21, Barcelona, Spain.

To Cite This Article: M Cristina de Almagro, The Use of Collagen Hydrolysates and Native Collagen in Osteoarthritis. 2020 - 7(6). AJBSR. MS.ID.001217. DOI: 10.34297/AJBSR.2020.07.001217.

Received: March 04, 2020; Published: March 06, 2020

\begin{abstract}
Osteoarthritis is a chronic degenerative disease that dampens the quality of life of those who suffer it. Conventional treatments are palliative, they are targeted to reduce joint pain. However, more efforts are needed to find new therapies that are able to positively affect or at least stop the course of this degenerative disease. Amongst the different therapeutic options that are being studied, collagen emerges as a good candidate due to its safety and preclinical and clinical evidence. Two different approaches have been taken for collagen: collagen hydrolysates and native collagen.

Research studies have shown that both types of collagen nutraceuticals are effective in reducing osteoarthritis pain, in animal models and in human clinical trials, however through different mechanisms of action. Hydrolyzed collagen can be absorbed and reach the target site where collagen synthesis is needed, while native collagen, which is poorly absorbed, could work through a mechanism of oral tolerance induction. Thus, collagen represents an interesting therapeutic option to improve osteoarthritis.
\end{abstract}

Keywords: Osteoarthritis; Collagen; Collagen Hydrolysate; Native Collagen; Undenatured Collagen; Dietary Supplement; Nutraceutical

Abbreviations: CH: Collagen Hydrolysate

\section{Introduction}

Osteoarthritis is a chronic degenerative joint disease, where the cartilage progressively gets degraded. This process is accompanied by a certain degree of inflammation, leading to pain and a reduced quality of life.

Longer life expectancy and an increasing aging population has brought up the importance of this disease, since its numbers are rising and is expected to continue doing so. Nowadays, there are several drugs that can be used for osteoarthritis, but they are mainly effective reducing pain but not improving the underneath cause, cartilage destruction. In an attempt to improve cartilage destruction, different alternative therapies have been used, among them, nutraceuticals such as chondroitin sulphate, glucosamine and hyaluronic acid [1]. However, lately a new dietary supplement to improve joint health has gained strength, collagen. Cartilage is made of $10 \%$ chondrocytes and $90 \%$ extracellular matrix, which is $60-70 \%$ water and the rest is organic material. In the cartilage organic material, the main protein found is collagen Type II.

\section{Collagen}

Collagen is the most abundant protein in mammals. It is mainly found as part of the extracellular matrix in connective tissues such as cartilage, tendons, ligaments, bones, skin, etc. It is a big protein, of approximately $400 \mathrm{KDa}$, with a complex structure due to its quaternary structure formed by a triple-helix. Its protein sequence is also special, since it is unusually rich in the amino acids hydroxyproline, proline and glycine, making the motif Gly-Pro-Hyp a hallmark of its sequence.

\section{Hydrolyzed Collagen}

Collagen hydrolysate $(\mathrm{CH})$ is the product of the enzymatic hydrolysis of native collagen to obtain smaller peptides. The rationality to hydrolyze collagen is not only to improve its solubility but also to improve its bioavailability. Due to its high molecular weight and triple-helix structure, absorption of orally ingested native collagen is scarce. The absorption of $\mathrm{CH}$ has been proven in animal models as well as in human studies $[2,3]$. A study where 
different doses of $\mathrm{CH}$ were given to healthy volunteers showed that blood hydroxyproline levels increased in a dose-dependent manner $[4,5]$. And noteworthy, hydroxyproline was found in blood not just as a free amino acid, but also in the form of small peptides [6]. In a mice study where the animals were fed radiolabeled (14)C-gelatin hydrolysate or $14(\mathrm{C})$-proline, $95 \%$ of the gelatin hydrolysate was absorbed [7]. Interestingly, in cartilage, the radioactivity accumulation was higher in those animals that consumed gelatin hydrolysate.

The efficacy of $\mathrm{CH}$ in osteoarthritis has been studied in human clinical trials. In one randomized, double-blind, controlled multicenter trial, 250 subjects with knee osteoarthritis were given $10 \mathrm{~g}$ of $\mathrm{CH}$ daily for 6 months. At the end of the study significant pain improvement was observed in the visual analogue scales and the WOMAC subscale [8]. Other clinical trials also showed reduction in joint pain upon $\mathrm{CH}$ daily consumption for periods ranging from 3 months to one year. In all these clinical trials an improvement in the WOMAC index as well as other indexes of joint functionality and discomfort were observed [9-13].

In animal models, studies of $\mathrm{CH}$ oral administration lead to the demonstration of $\mathrm{CH}$ pain reduction in osteoarthritis-like lesions as well as the improvement of other disease markers such as the increase in cartilage area, number of chondrocytes, typeII collagen levels and the decrease in MMP-13 and CTX-II (Cross Linked C-Telopeptide of Type II Collagen, a biomarker of cartilage degradation) levels [14-17].

\section{Native Collagen}

Another area of study is the administration of native collagen instead of $\mathrm{CH}$. In this case, the mechanism of action is believed to be different to that of $\mathrm{CH}$, where $\mathrm{CH}$ could function as bioactive peptides or as a source of the specific amino acids needed to build collagen. Due to the low absorption rate of native collagen, the mechanism of action is believed to take place in the gastrointestinal track. The hypothesis is that, in a similar way as food proteins, native collagen will induce oral tolerance [18]. Native collagen would modulate the response of the gut immune system, gut-associated lymphoid tissue (GALT), to switch to a tolerogenic response against collagen. This will train the immune system not to recognize collagen joint degradation products as DAMPs (Damage-associated molecular patterns) and avoid joint damage produced by the immune system activation. In two rat studies where osteoarthritis was induced by MIA intra-articular injection, native collagen reduced limb pain in the animals. In one study they also observed a decrease in plasma and urinary CTX-II [19]. And in the other rat study there was a decrease in degraded C2C (a biomarker of cartilage degradation) as well as in the inflammatory cytokines IL-1 $\beta$ and TNF $\alpha$ [20].

In humans the efficacy has been proven in some clinical trials. In one clinical trial, where the effect of glucosamine and chondroitin sulfate with or without collagen was assessed, the addition of collagen improved the urinary levels of CTX-I and CTX-II as well as the hand radiological score after 1 year of treatment [21]. Another clinical study where knee osteoarthritis patients consumed for 6 months undenatured collagen showed a reduction in WOMAC score improving pain and joint functionality [22]. Moreover, a clinical trial in which collagen was given with acetaminophen, after 3 months of treatment the group that consumed collagen had a greater improvement in WOMAC index, gaining functionality in comparison with the group that took only acetaminophen [23].

\section{Conclusion}

Efforts are being made to find new therapeutic options that can not only reduce osteoarthritis pain, but also improve the disease course. Nutraceuticals such as collagen are good candidates due to their safety profile and studies that show osteoarthritis improvement. However, more studies are needed to clarify collagen mechanism of action and larger clinical trials should bring more evidence of the role of collagen supplements in osteoarthritis.

\section{Conflict of Interest}

The author is an employee of Laboratorios Ordesa S.L.

\section{References}

1. Ragle RL, Sawitzke AD (2012) Nutraceuticals in the Management of Osteoarthritis. Drugs Aging 29(9): 717-731.

2. Kawaguchi T, Nanbu PN, Kurokawa M (2012) Distribution of prolylhydroxyproline and its metabolites after oral administration in rats. Biol Pharm Bull 35(3): 422-427.

3. Iwai K, Hasegawa T, Taguchi Y, Morimatsu F, Sato K, et al. (2005) Identification of food-derived collagen peptides in human blood after oral ingestion of gelatin hydrolysates. J Agric Food Chem 53(16): 65316536.

4. Shigemura Y, Kubomura D, Sato Y, Sato K (2014) Dose-dependent changes in the levels of free and peptide forms of hydroxyproline in human plasma after collagen hydrolysate ingestion. Food Chem 159: 328-332.

5. Skov K, Oxfeldt M, Thøgersen R, Hansen M, Bertram HC (2019) Enzymatic Hydrolysis of a Collagen Hydrolysate Enhances Postprandial Absorption Rate-A Randomized Controlled Trial. Nutrients 11(5): 1064.

6. Ichikawa S, Morifuji M, Ohara H, Matsumoto H, Takeuchi Y, et al. (2010) Hydroxyproline-containing dipeptides and tripeptides quantified at high concentration in human blood after oral administration of gelatin hydrolysate. Int J Food Sci Nutr 61(1): 52-60.

7. Oesser S, Adam M, Babel W, Seifert J (1999) Oral administration of (14) $\mathrm{C}$ labeled gelatin hydrolysate leads to an accumulation of radioactivity in cartilage of mice (C57/BL). J Nutr 129(10): 1891-1895.

8. Benito Ruiz P, Camacho Zambrano MM, Carrillo Arcentales JN, Mestanza Peralta MA, Vallejo Flores CA, et al. (2009) A randomized controlled trial on the efficacy and safety of a food ingredient, collagen hydrolysate, for improving joint comfort. Int J Food Sci Nutr 60 (Suppl 2): 99-113.

9. McAlindon TE, Nuite M, Krishnan N, Ruthazer R, Price LL, et al. (2011) Change in knee osteoarthritis cartilage detected by delayed gadolinium enhanced magnetic resonance imaging following treatment with collagen hydrolysate: a pilot randomized controlled trial. Osteoarthritis Cartilage 19(4): 399-405.

10. Trč T, Bohmová J (2011) Efficacy and tolerance of enzymatic hydrolysed collagen (EHC) vs. glucosamine sulphate (GS) in the treatment of knee osteoarthritis (KOA). Int Orthop 35(3): 341-348. 
11. Jiang J, Yu S, Huang QR, Zhang XL, Zhang CQ et al. (2014) Collagen peptides improve knee osteoarthritis in elderly women - a 6-month randomized, double-blind, placebo-controlled study. Agro Food Industry Hi Tech 25(2): 19-23.

12. Bruyère O, Zegels B, Leonori L, Rabenda V, Janssen A, et al. (2012) Effect of collagen hydrolysate in articular pain: a 6-month randomized, doubleblind, placebo-controlled study. Complement Ther Med 20(3): 124-130.

13. Carpenter RL, Peel, JB, Carpenter MR, Lowndes J, Angelopoulos TJ, et al. (2005) Effectiveness of a collagen hydrolysate-based nutritional supplement on the level of joint pain, range of motion and muscle function in individuals with mild osteoarthritis of the knee: a randomized clinical trial. Ann Rheum Dis Sup 3: 1544-1545.

14. Dar QA, Schott EM, Catheline SE, Maynard RD, Liu Z, et al. (2017) Daily oral consumption of hydrolyzed type 1 collagen is chondroprotective and anti-inflammatory in murine posttraumatic osteoarthritis. PLoS One 12(4): e0174705.

15. Isaka S, Someya A, Nakamura S, Naito K, Nozawa M, et al. (2017) Evaluation of the effect of oral administration of collagen peptides on an experimental rat osteoarthritis model. Exp Ther Med 13(6): 2699-2706.

16. Ohara H, Iida H, Ito K, Takeuchi Y, Nomura Y (2010) Effects of Pro-Hyp, a collagen hydrolysate-derived peptide, on hyaluronic acid synthesis using in vitro cultured synovium cells and oral ingestion of collagen hydrolysates in a guinea pig model of osteoarthritis. Biosci Biotechnol Biochem 74(10): 2096-2099.

17. van de Water E, Oosterlinck M, Dumoulin M, Korthagen NM, van Weeren PR (2017) The preventive effects of two nutraceuticals on experimentally induced acute synovitis. Equine Vet J 49(4): 532-538.
18. Park KS, Park MJ, Cho ML, Kwok SK, Ju JH (2009) Type II collagen oral tolerance; mechanism and role in collagen-induced arthritis and rheumatoid arthritis. Mod Rheumatol 19(6): 581-589.

19. Di Cesare Mannelli L, Micheli L, Zanardelli M, Ghelardini C (2013) Low dose native type II collagen prevents pain in a rat osteoarthritis model. BMC Musculoskelet Disord 14: 228.

20. Di Cesare Mannelli L, Maresca M, Micheli L, Martin Puig D, Ghelardini C (2015) Low dose chicken native Type II collagen is active in a rat model of osteoarthritis. World Congress on Osteoporosis, Osteoarthritis and Musculoskeletal Diseases, Italy.

21. Scarpellini M, Lurati A, Vignati G, Marrazza MG, Telese F (2008) Biomarkers, type II collagen, glucosamine and chondroitin sulfate in osteoarthritis follow-up: the "Magenta osteoarthritis study". J Orthop Traumatol. 9(2): 81-87.

22. Lugo JP, Saiyed ZM, Lane NE (2016) Efficacy and tolerability of an undenatured type II collagen supplement in modulating knee osteoarthritis symptoms: a multicenter randomized, double-blind, placebo-controlled study. Nutr J 15: 14.

23. Bakilan F, Armagan O, Ozgen M, Tascioglu F, Bolluk O, et al. (2016) Effects of Native Type II Collagen Treatment on Knee Osteoarthritis: A Randomized Controlled Trial. Eurasian J Med 48(2): 95-101. 